Cahiers d'études africaines

209-210|2013

Masculin pluriel

\title{
Fils, frères, pères
}

Masculinités sous contrats, du nord à la capitale du Mali

Sons, Brothers, Fathers. Masculinity Under Contracts, From The North to The

Capital of Mali

\section{Monique Bertrand}

\section{OpenEdition}

\section{Journals}

Édition électronique

URL : http://journals.openedition.org/etudesafricaines/17348

DOI : $10.4000 /$ etudesafricaines. 17348

ISSN : 1777-5353

Éditeur

Éditions de l'EHESS

\section{Édition imprimée}

Date de publication : 28 mai 2013

Pagination : $323-344$

ISBN : 978-2-7132-2387-7

ISSN : 0008-0055

Référence électronique

Monique Bertrand, «Fils, frères, pères », Cahiers d'études africaines [En ligne], 209-210 | 2013, mis en ligne le 06 juin 2015, consulté le 15 juin 2020. URL : http://journals.openedition.org/etudesafricaines/ 17348 ; DOI : https://doi.org/10.4000/etudesafricaines.17348

Ce document a été généré automatiquement le 15 juin 2020

(c) Cahiers d'Études africaines 


\title{
Fils, frères, pères
}

\author{
Masculinités sous contrats, du nord à la capitale du Mali \\ Sons, Brothers, Fathers. Masculinity Under Contracts, From The North to The \\ Capital of Mali
}

\section{Monique Bertrand}

1 La montée en force de perspectives "genrées » de développement n'échappe pas au Mali à un centrage sur les femmes. Leur prise en compte comme un sujet collectif et global relève de deux lectures complémentaires: celle de l'accaparement des ressources, du numéraire à la parole publique, constitue l'argument général de la domination masculine, dans les lieux de la reproduction sociale comme dans ceux du travail et de la production; pragmatique, la formule de l'empowerment est aussi plus récente mais s'attache aussi bien aux citadines qu'aux villageoises, aux enjeux de la migration qu'aux perspectives locales de la citoyenneté (Bertrand 2011b).

2 L'analyse des marges d'autorité des hommes se déplace donc en suivant les marges d'action déniées aux femmes puis revendiquées par ou pour elles. Dans l'espace public, la masculinité s'ajuste ainsi à la visibilité que prennent les secondes selon des ciblages institutionnels, des labels de reconnaissance ou de participation, ou des lignes de crédits fléchés, articulés aux agendas internationaux. De «bonnes pratiques» fragmentaires s'adressent tantôt à des migrantes saisonnières, tantôt à de jeunes déscolarisées, à des veuves en quête de produits à valoriser, des actives à stabiliser par le microcrédit, etc. De tels formats d'intervention sociale produisent une vision tronçonnée de la société malienne dans laquelle les « hommes jeunes » sont eux-mêmes invités à soutenir les meilleures initiatives... de soutien aux filles. Non sans chassécroisé avec la recommandation "post-conflit» faite aux femmes du Nord Mali à s'engager dans la pacification des rapports interethniques durant les années 2000, la masculinité se trouve ainsi mobilisée en catégories finalisées "to achieve gender equality $»^{1}$.

Des soubassements de l'hégémonie masculine aux paris de co-émancipation des cadets sociaux, le renversement de perspective n'est qu'apparent. L'expérience post-apartheid de l'Afrique du Sud invite en effet à re-historiciser la question de la domination 
masculine sur le triptyque race/classe/sexualité, en soulignant l'empilement des violences, l'intrication d'agressivités reçues par les hommes et infligées aux femmes dans un continuum de rapports économiques et privés, sportifs et politiques (Morrell 1998). Dans la société marocaine, de même, la masculinité cherche à se maintenir comme dominante sur le contrôle sexuel des filles et le contrôle de la sexualité féminine dans le mariage, en composant avec des changements évidents de la place des femmes « entre tradition, modernité et intégrisme » (Dialmy 2009).

4 L'approche retenue ici dans le cas de Bamako prend acte de ces attendus de "participation» au développement, et de ce versant des rapports amoureux et des identités sexuelles entre hommes et femmes (Dumestre \& Touré 1998; Broqua \& Eboko 2009). Mais elle s'inscrit dans l'étude d'un contrat social urbain encore structuré par les rapports intergénérationnels. L'analyse s'attache alors à un enjeu matériel et ordinaire de la pratique de la ville, résidentiel, dans lequel le partage de tâches est "genré » : à l'homme l'appropriation du sol et l'édification des murs de la maison ou le paiement d'un loyer; à la ménagère le soin quotidien du foyer ${ }^{2}$. Écho des rapports migrantscitadins dans la fabrique du vivre ensemble urbain (Gibbal 1974), l'habitation est aussi présentée comme étant lieu de violences et de civilités rendues incertaines (Janin 2003). Au-delà des ressources marchandes qu'elle requiert en ville, sa responsabilité engage la capacité des hommes, d'une génération à l'autre, à «tenir rang " dans les voisinages d'agglomérations en pleine expansion. Dans cette perspective, les affects exprimés dans une gamme de réussites et d'échecs s'attachent moins aux techniques du corps, aux expressions de la virilité qu'aux expériences de la cohabitation.

5 Le pluriel s'impose en effet, même en se restreignant comme ici aux couches populaires de la société bamakoise. Car l'habitation implique de suivre les hommes d'abord comme « logés », puis comme « logeurs » dans une responsabilité qui les démarque des femmes et les relie en même temps à elles. Que ce soit dans la référence bambara à la jatigiya le fait de se porter hôte hébergeant d'« étrangers » à la localité, selon une pratique en réalité banale au Mali - ou dans les quartiers d'immigrés musulmans d'Afrique de l'Ouest, l'urbanité s'illustre dans la propension des aînés sociaux à investir lieux et sociabilités au regard de normes d'entregent (Vuarin 1994) qui sont apprises d'abord dans l'habitat de cour. Depuis la colonisation, la résidence urbaine maintient la fonction de réception de visiteurs temporaires et d'accumulation de résidents plus durables, les premiers et les seconds étant communément désignés comme « hébergés » sous le toit d'un homme.

6 De la cohabitation d'épouses et d'enfants à celle de nouvelles générations mariées, de l'accueil de jeunes confiés aux hommes à celui de dépendants mobilisés par les femmes, cet habitat n'échappe pas à la dégradation des conditions de vie en ville dans les trente dernières années. Pourtant, l'idée qu'une femme reste à tous âges hébergée par un homme, tuteur puis mari, le fait qu'elle échappe comme adulte à la définition du mode d'occupation du logement de la plupart des ménages, relèvent tant du sens commun que de l'approche statistique des catégories résidentielles ${ }^{3}$. Ce sont bien deux individuations qui se jouent dans la ville: pour les hommes, l'hébergement ne représentera qu'un moment du cycle de vie, plus ou moins prolongé de l'enfance aux premières étapes du parcours urbain; au contraire, l'hébergement restera un marqueur à vie de l'infériorité sociale et économique des citadines dans la condition du mariage. Leur désignation chronique comme hébergées n'est démentie ni quand l'épouse commence à assumer la charge du ménage, ni par la percée de certaines 
d'entre elles sur le marché des parcelles à bâtir (Bertrand 2001). Même bailleuses de maisons, les citadines restent "logées par le mari", non informées sur les coûts des terrains, des constructions et des loyers dès lors qu'ils sont «l'affaire du mari », et dédouanées de la classification des tenures des logements. Le fait d'en compter aujourd'hui $12 \%$ parmi les référents des ménages reste associé à un temps anormalement long d'isolement par divorce ou veuvage.

7 Pour se placer du point de vue des hommes, la présente analyse s'appuie sur le corpus «Ego Fratries Générations » d'entretiens menés en 2008 et 2009 auprès de quinze natifs des régions de Gao et Tombouctou au Nord du Mali, âgés de soixante ans et plus, précisément sur les récits de vie et de famille d'un groupe de maçons, boutiquiers de quartier, jardinier, blanchisseur-cultivateur, cuisinier, plus ou moins encore en activité à l'enquête. Leurs expériences de cohabitations sont racontées comme fils, frères, pères et tuteurs, et sont suivies selon des parcours de migration et d'insertion urbaines souvent précaires et chaotiques, ajustés aux trajectoires matrimoniales, aux vies de travail, aux relations avec les communautés d'origine. S'ajoutent à ces récits individués, les inventaires relatifs à trois générations définissant l'espace de vie familial d'« ego »: ses ascendants et tuteurs à l'enfance, d'une part, ses frères et sœurs, et pairs en génération, d'autre part, sa descendance enfin éduquée mais aussi confiée et reçue pour un nombre significatif d'années. C'est donc en suivant leurs lieux de vie, cadres d'apprentissage, de transmission ou de mises à distance de normes sexuées d'habitation, que des hommes sont situés dans leurs pratiques et leurs représentations de l'hébergement.

8 Le recueil d'expériences donne en effet de nombreux échos des codes de l'honneur et de la honte associés à la résidence en ville (Bouju 2009) : désignations explicites et marques silencieuses, non-dits, ton réservé ou formules convenues s'en remettant à la volonté divine, parole interrompue et relayée par un geste d'impuissance. La pratique d'hébergement tire-t-elle alors la masculinité vers une hégémonie reformulée ou vers les frustrations d'une marginalisation en milieu populaire (Connell 1995) ? En matière d'habiter, faut-il ici aussi s'inspirer d'une sociologie des men's studies, dans la lignée de mouvements anti-racistes et féministes, de reconnaissances gay et bisexuelles (GormanMurray 2008) ? Plutôt que de partir de références occidentales et d'environnements consuméristes, on relativisera ici la suprématie masculine en déroulant une temporalité intergénérationnelle (Oppong 2006; Antoine 2007) et en s'attachant aux effets d'une urbanisation privée de croissance économique (Bertrand 2011a). Entre des fils entrants dans la vie adulte et des pères vieillissants, "qui héberge qui ?». Les normes de cohabitation résidentielle se maintiennent-elles à l'identique dans un groupe homogène en âges et origines régionales? Selon quels écarts de pratiques et quelles lignes de conflits? C'est bien relativement à des hommes nés en brousse dans les années 1940, et à leur relève née en ville à partir des années 1970 que se situent ici les capacités et les épreuves de l'hébergement.

\section{D’hébergé à hébergeant : masculinité en mouvements dans une triple transition}

9 Devenir hébergeant non seulement s'apprend, mais manifeste une forme de performance chez les hommes interrogés. Chacun s'en prévaut comparativement à ce qui est rapporté d'autres hommes, avec la fierté de l'assumer ou le regret de ses 
carences. Mais il convient de relier historiquement à la colonisation le principe d' « asseoir » la maisonnée, d'y consacrer des « réalisations », d'ancrer territorialement la résidence et ses cohabitations dans un cadre marchand. Non pas que les ménages des migrants soient devenus nucléaires à Bamako ${ }^{5}$, mais parce qu'ils ont bien rompu avec des modes précoloniaux de reproduction domestique et leurs survivances associées aux rapports maîtres/captifs (Olivier de Sardan 1984). C'est dans un principe de localité, articulé aux dynamiques migratoires contemporaines, que se recompose l'habiter. Dans les mémoires familiales reconstituées par l'enquête, il se démarque alors des circulations instables de jeunes actifs, d'une part, d'essaimages incertains d'autre part, rappelés quand les chefs de canton coloniaux reconvertissaient une accumulation guerrière en stratégies d'accaparement foncier.

Mais une fois admise la fixation normative de la résidence, c'est sur sa capacité à y faire cohabiter plusieurs générations et à y relier des lieux catégorisés comme "d'origine ", de «transit », ou d'« installation », dans un mouvement finalisé par le chef de famille, que se graduent les performances des logeurs. L'approche longitudinale retenue permet précisément d'en séparer les responsabilités: d'un côté ce qui est présenté comme invariant des habitus d'entregent; de l'autre, des éléments plus circonstanciés propres à une génération, à un âge de la vie, ou à un moment d'insertion sur les marchés fonciers de la grande ville. Car l'ajustement structurel de l'économie malienne, depuis 1983, puis les orientations néolibérales de la Troisième République, depuis 1992, ont montré leurs effets sociaux à Bamako: retard d'émancipation des jeunes générations, différence de taille des ménages propriétaires et locataires, nouveaux arbitrages entre époux pour la prise en charge de cadets familiaux (Ouédraogo \& Piché 1995 ; Bertrand 2004).

\section{Du village à la ville, de logé à logeur}

11 La transition migratoire affecte en premier lieu les codes sexués d'habitation de familles qui se redistribuent $d u$ nord au sud du Mali, et de contextes agraires vulnérables dans la Boucle du Niger, à la capitale, perçue comme phare de la modernité. Loin d'une translation simple, les parcours d'hommes sont faits de fréquents heurts internationaux et s'inscrivent dans une précarité économique prégnante. À leur terme, Bamako compte 1,8 million d'habitants. Son agglomération diffère non seulement des milieux délaissés par la migration, mais de la ville de moins de 400000 habitants abordée 30 à 40 ans auparavant, au début du cursus résidentiel urbain.

Le rapport au logement apparaît ainsi construit selon différents types de localités puis au fil de déménagements centrifuges en agglomération, des vieux quartiers centraux aux marges recherchées pour leurs coûts fonciers et locatifs moindres. Son déroulement biographique est ainsi gradué en âge : le passage par un tuteur, désigné par le père ou recherché à son décès, justifie la plus grande jeunesse moyenne (16 ans) des étapes de vie dans les régions du Soudan colonial, devenu Mali ; «l'aventure » de travail en Afrique de l'Ouest - Ghana, Niger, Côte-d'Ivoire - marque l'émancipation des jeunes gens (22 ans) ; la réorientation migratoire des adultes, décidés à se fixer au Mali et à établir une famille à distance du Nord, fonde la moyenne la plus élevée (38 ans) pour les étapes de mobilité à Bamako. C'est donc à l'expérience de sérieux changements de contextes que se rodent la demande d'hébergement et sa transformation en offre. 
13 Aux phases de migration interrégionale, la gamme de logeurs sollicités pour la formation des jeunes dépasse encore le lien familial d'origine, et laisse subsister des réseaux sociaux maraboutiques, de classes d'âge des ascendants, voire des solutions improvisées dans les milieux urbains plus cosmopolites. Ces occasions tendront à disparaître plus tard, quand " ego » parvenu lui-même à l'âge adulte, n'acceptera de confier ses propres enfants qu'à des parents proches. Dans la capitale malienne, l'évolution de l'accueil se mesure en particulier dans le fait que bien des hommes nés dans d'autres régions du Mali se disent avoir été redevables d'aînés de leur famille à leur arrivée en ville, puis logeurs de leurs propres cadets mais « oubliés » quand ceux-ci se réorientaient vers de nouvelles destinations migratoires ou vers un statut de travailleur salarié. Gratitudes et ingratitudes structurent ainsi les transmissions intergénérationnelles. Il n'empêche que la distance prise par les hommes avec les terroirs d'origine, au profit de subsistances nouvelles, a déclenché un cycle d'obligations qui suscite des retours de dettes à chaque étape de vie. Même quand l'hébergement offert est précaire, comme dans une boutique de quartier assurant à la fois un emploi temporaire et le coucher, il reste associé à « la chance » du logeur et à toutes sortes de débrouilles du citadin pour ses obligés.

14 C'est donc bien Bamako qui concentre près de la moitié des étapes de mobilité enregistrées dans les parcours des migrants du Nord, et la quasi-totalité de leur vie d'adultes. Dès lors la constitution puis l'élargissement du ménage, par mariages successifs ou cumulés ${ }^{6}$, naissances et accueils durables, démultiplient les catégories et les transitions résidentielles de ces hommes : le passage d'un hébergement "à titre gratuit » à un autre se fait en moyenne à 33 ans; la sortie de l'hébergement prend place à 39 ans pour la location et à 41 ans pour la propriété.

15 Le contrat social de redistribution des bénéfices de la migration, à l'égard de la famille à l'origine, se double alors du contrat éducatif dont sont redevables les chefs de famille à l'égard de leur descendance propre et de cadets issus de leurs fratries. Pour ces derniers, la demande d'hébergement à Bamako se justifie par les ressources en travail et en formation, plus souvent dans l'apprentissage qu'à l'école, qui sont jugées meilleures qu'au Nord. S'y ajoutent des perspectives de traitements médicaux qu'offrent aux villageois l'interruption saisonnière du travail agricole. À défaut d'argent ou de dons en nature envoyés au village, les nuitées offertes dans l'environnement de ces ressources déplacent les normes de redistribution sur la rente urbaine. Dans une réelle continuité postcoloniale, elle assure l'hébergeant de « l'échange de nouvelles » pour ses propres stratégies matrimoniales, de prestations en travail pour la maintenance de son logement ou pour ses activités professionnelles.

Une fois passées les formules de logement bricolées dans les premiers temps des mariages, ménager une capacité d'accueil dépassant le noyau conjugal se présente même comme le moteur de la mobilité résidentielle pendant les années (17 en moyenne) qui séparent l'arrivée à Bamako et l'installation dans le dernier logement visité en 2009. Un boutiquier en retraite justifie ainsi son déménagement du centre à la périphérie urbaine dans les années 1980 par la nécessité de disposer « d'une ou de deux chambres pour préserver le lien parental et accueillir les étrangers qui viendront du village ».

17 Mais quand des visiteurs se transforment en hébergés durables, et des gens de passage en débiteurs tenaces d'une année à l'autre, "aider les mêmes qui vous ont aidés » 
rejoint les arguments plus intimes de transformation d'un bien marchand en patrimoine d'homme.

\section{Transition démographique : descendance directe et nouvelles générations confiées}

Malgré des trajectoires matrimoniales déjà engagées dans les régions d'origine, l'installation des hommes du Nord à Bamako coïncide en effet largement avec la constitution de la descendance. Un mécanicien revient ainsi sur la décennie des années 1980 qui amorce à la fois la transition démographique en ville et l'ajustement structurel dans le pays, et qui coïncide pour son ménage polygame au déménagement vécu parmi d'autres comme le plus décisif. D'une location source de «bagarres quotidiennes entre les deux femmes ", on passe à la construction de la maison en périphérie non lotie de la ville qui restaure la dignité du mari et le pose en pionnier d'un nouveau peuplement urbain. Peu importe que la première épouse s'éloigne finalement après ce temps d'accès à la propriété, c'est le moment perçu comme « changement d'époque » à la fois pour la ville et pour ses habitants déterminés à "rester le maître dans la famille ", capables de réguler les troubles d'une cohabitation subie.

Cette première phase de changements familiaux réduit la mortalité sans encore réduire la natalité. Couplée avec la multiplicité des unions et une fécondité concurrentielle des épouses, elle confère aux hommes une moyenne de neuf enfants. Elle étale surtout les naissances jusqu'à un âge avancé des pères, et conduit aujourd'hui à un sérieux écart de génération entre ceux-ci et leurs enfants encore en vie, dont la moyenne d'âge n'est que de vingt ans à l'enquête. À l'heure des retraites, la descendance directe n'est en situation ni professionnelle ni matrimoniale ni résidentielle de contribuer aux charges de la maisonnée du «vieux », de se préparer elle-même à en quitter le ménage, et de pourvoir aux besoins d'une fratrie plus jeune.

Le creux temporaire de descendance directe a surtout produit un appel d'air pour l'hébergement de jeunes en formation ou en attente de mariage. Cette descendance indirecte se partage en fait entre des enfants confiés que les hommes ont pris en charge au début de leur vie d'adulte, dont les classes d'âge (de 20 à 40 ans) dépassent celles de ses propres enfants; et ceux qui viennent plus tard dans la maturité, en relais de leur descendance directe, en particulier des petits-enfants laissés par leurs parents. Les premiers ont rodé les codes sexués de répartition des enfants dans l'espace disponible, sous l'autorité du père de substitution; les seconds prennent place davantage « sous la surveillance des mamans ».

21 À l'étalement de ces pratiques d'accueil, que ponctuent parfois plusieurs déménagements intermédiaires, s'ajoutent enfin des retours de résidence après divorce. Il s'agit de filles éduquées d'abord loin du père après la séparation de leurs parents, mais aussi, et de plus en plus, de filles elles-mêmes mal mariées après avoir grandi sous le toit paternel. La cohabitation se complexifie en effet du fait de ruptures d'unions sur plusieurs générations : celles de l'hébergeant peuvent désolidariser une partie de sa descendance du reste de la maisonnée; celles de ses filles diversifient les profils et les temporalités d'accueil.

22 Avec l'avancée en âge, les plus grands logeurs ont donc surajouté des femmes à leur hébergement: signe des déconnexions de l'habitation et du travail en ville, et d'un sérieux changement d'enjeu de la gouvernance domestique. Les résidentes temporaires 
multiplient les chassés-croisés selon des « recrutements » de plus en plus hétérogènes en âges (avant/après mariage), en parentés et lieux d'origine (non plus restreints au Nord mais essaimés jusqu'en migration internationale), en intentions et intérêts à solliciter ainsi le toit des hommes (visites «pour voir ", gestion d'imprévus, fécondité hors mariage, besoins de divorces). Qu'ils soient géniteurs ou tuteurs de remplacement, ceux-ci ressortent à la fois bonifiés et affectés de ce mélange de secours accordés aux filles reçues d'une "sœur » ou d'une épouse, aux élèves à scolariser, à des adultes non encore ou mal mariées. Les prises en charge " par pitié » annoncent un contrôle devenu incertain de la sexualité des filles, à commencer par celles des logeurs, ce qui devient une composante même de la transition démographique. Dans l'échantillon des migrants $\mathrm{du}$ Nord, le seul père d'un enfant naturel, un soldat reconverti restaurateur, se retrouve, à l'enquête, gardien de huit petits-enfants dont sept naturels reçus d'une fille et de trois garçons non mariés parmi ses enfants légitimes. Le commentaire qu'il mène de ses deux derniers enfants encore cohabitants est éloquent: d'un côté un fils "vagabond » dont on ne parvient pas à capitaliser les expériences d'apprentissage : de l'autre, une mère célibataire détournée des normes de sortie de la maison paternelle, mais garantissant finalement mieux, par ses activités de commerce, un retour sur investissement pour les « contributions à la maison » couvertes par les femmes.

\section{Transition métropolitaine : l'accès aux ressources urbaines}

Dans les deux dernières décennies, non seulement la vie en ville mais son étalement d'ampleur métropolitaine démultiplient les coûts d'accès au travail: logement, transports, services marchands, mais aussi offres scolaires et sanitaires que la Troisième République malienne a privatisées. L'épanouissement normatif des ménages citadins, le standing et la renommée de leurs référents sont compromis. En ouvrant à la location quelques pièces de leurs maisons déjà chargées, des propriétaires sont vus comme sacrifiant leur parentèle d'origine, en même temps qu'ils assurent un complément de revenus pour le quotidien du plus petit cercle familial. C'est bien d'une transition urbaine qu'il s'agit, du fait d'une difficulté croissante à sécuriser le statut d'hébergeant dans un accès de plus en plus sélectif aux ressources de la capitale.

Car après les deux dévaluations monétaires de 1984 et 1994, la rente urbaine ne se mobilise ni ne circule plus aussi aisément dans les familles. La dégradation des pouvoirs d'achat est d'autant plus sévère qu'elle frappe des migrants dont les activités professionnelles sont instables dans le secteur informel. Celles-ci s'achèvent sans couverture sociale, sans que le vieillissement ait été anticipé par un investissement scolaire conséquent pour les fils ou par le mariage gratifiant de filles dans d'autres environnements urbains que ceux des pères.

L'accès à la propriété qui constitue la cheville ouvrière des stratégies d'accumulation de dépendants sociaux, devient surtout incertain aux âges avancés où les hommes du Nord se redistribuent vers les périphéries bamakoises. Leur inscription dans les concurrences foncières de la capitale malienne apparaît trop tardive et les montre locataires ballottés d'une cour à l'autre jusqu'à cinquante ans, un âge moyen " anormalement » élevé dans les cursus urbains. Ce retard est fatal à près d'un sur deux, en les plaçant face à des coûts du sol nettement accrus. Non seulement les lotissements administrés leur sont inabordables, mais les prix ont explosé dans les quartiers spontanés qui font l'objet de régularisations dans les années 1990. Au total, 
les « vieux » du Nord se partagent entre propriétaires et locataires, dans un rapport numérique comparable à celui qui marque les ménages bamakois - $46 \%$ de propriétaires, 44 \% de locataires - dès le recensement de 1987.

Chez les propriétaires, l'hébergement de cadets sociaux se montre désormais sous condition, sinon dissuasif. Si la condition n'est pas énoncée explicitement comme financière, ce qui à l'égard de parents paysans vaudrait refus frontal, elle conduit à des impossibilités de rester durablement, elle fluidifie le roulement des visiteurs et répartit la charge de l'hébergement dans le temps. Des sollicitations sont également reportées sur un autre logeur et mettent de fait certains frères à distance en dissimulant une réserve catégorique.

Quant aux hommes restés locataires au dernier logement occupé, même des transactions incertaines, même l'acquisition de parcelles éloignées ou de terrains en fortes pentes n'offrent plus assez d'opportunités: la course au sol et sa gestion municipale clientéliste multiplient sélections et évictions politico-marchandes dans les deux dernières décennies. À la forte instabilité matrimoniale des propriétaires répond donc une monogamie de précarité subie comme honteuse par les locataires. Le différentiel de taille de leurs ménages est plus que jamais démonstratif pour un groupe de mêmes origines et classes d'âge. La pauvreté attachée aux seconds prend de plus une tournure cumulative quand la location s'inscrit dans un habitat de cour non sécurisé, menacé de casses - bornage de parcelles, ouverture de voies - par les restructurations urbaines des années 1990. Dans ce cas, le logement est réduit à la portion congrue d'une ou deux pièces sans terrasse, et son occupant n'enregistre ni visite prolongée ni demande de confiage.

Aux écarts de composition des ménages s'ajoutent en effet ceux du flux de visiteurs. Le vocabulaire de la capacité versus la privation qualifie ainsi les hommes en s'ajustant à l'opposition de plus en plus sensible des propriétaires sédentarisés et de locataires nomades en ville. Il recycle volontiers le lexique bien rodé, sur les terrains domestiques et politiques, du pouvoir (faama en bambara) et du défaut d'entregents (faantan). La masculinité se trouve prise à défaut de toujours répondre aux sollicitations depuis les régions d'origine, et de se prévaloir de l'hospitalité. Dans un cas de dénuement sévère, la honte se dissimule derrière un discours normé sur la prise en charge des démunis dans la communauté des musulmans, quand toute l'histoire résidentielle du "vieux ", de son premier hébergement à plusieurs évictions locatives, relève en réalité de sa prise en charge par des tiers non familiaux. Humiliante à son âge quand elle est reçue au lieu d'être donnée, la « solidarité » est enjolivée comme gratification à l'ombre de la mosquée qui lui a donné une place de muezzin. Mais elle tait surtout l'impossibilité à revenir chez soi les mains vides ou à solliciter ses enfants pour manger.

Mais de faux-semblants convenus en retenues communautaires, la responsabilité d'homme glisse vers l'aveu direct de formules maigres d'hébergement ou d'incapacités durables: des parents qui dorment sous la véranda, des promesses de visite guère concrétisées, les hébergés d'une nuit qui s'empressent de filer à leurs affaires, et ceux qu'on envoie dormir à l'extérieur parce que « $\mathrm{y}$ a plus de place là-bas que ici ».

D'abord bonifiée par la migration, la masculinité se montre étriquée et marginalisée dans les périphéries urbaines. Elle est mise sans "pudeur $»^{7}$ dans les mots de la pauvreté, elle fait contrepoint à la masculinité installée, chargée, des grands hébergeants. Ses contradictions sont désormais commentées entre hommes de même origine sociale et régionale. Le vocabulaire de la frustration économique décharge « la 
honte devant les femmes et les enfants" des connotations morales de l'indignité personnelle, et la reporte sur les détresses de l'époque. Les termes de « capacités » et de "lutte contre la pauvreté ", que relaient campagnes électorales et oNG, contribuent à cette déculpabilisation et conduisent aux aveux de faillites professionnelles. Même ceux qui ne s'autorisent pas à décrire crûment leur « malchance ", ni à échanger sur ce point avec les générations plus jeunes, ne peuvent refouler les commentaires qu'elle suscite dans les sociabilités de voisinage: "Dieu est grand! Mais quand on est pauvre on peut pas se reposer. »

31 Attribut matériel et prérogative décisionnelle des hommes, l'hébergement suscite finalement des représentations graduées, défendues à chaque âge de la vie en reliant différents milieux de vie. Il relie aussi différentes générations familiales en vertu de contrats d'obéissance et de redistribution qui se renégocient au fil de ces expériences migratoires et urbaines.

\section{Masculinités sous tension : mobilisation et déstabilisation des références normatives}

Par-delà le déroulement des vies dans l'étalement des villes, qui semble dichotomiser les masculinités hégémonique et marginalisée, d'autres influences familiales configurent le comportement des hommes de manière plus fine. De véritables tensions discursives marquent ainsi les hébergeants potentiels, avérés ou désavoués, comme fils, frères et pères.

\section{Tels fils tel père ? Respect, fuite, inconscience}

Dans les histoires familiales, l'autorité des hommes s'exprime par le contrôle de la mobilité, des ressources drainées par le travail, et des engagements matrimoniaux des cadets familiaux. Sur ce fond commun de normes, l'expérience de chacun apporte validations et transgressions pratiques : ici le respect confronté à la désobéissance, cette dernière étant volontiers traduite en "trahison "; là l'escapisme repenti, la correction revendiquée de conduites immatures, auxquels est opposé celui qui "s'entête" dans son irresponsabilité. C'est en revenant sur leur passé de fils et de frères, que les pères vieillis d'aujourd'hui graduent la valeur des hommes encore jeunes auxquels ils étaient ou sont encore censés transmettre les bonnes règles de conduite.

Sur le chapitre de l'obéissance, les expériences des hommes engagent directement la relation père/fils. Mais elles se montrent plurielles une fois rappelée la référence à la tradition. Dans un cas, le récit familial relie un fils obéissant (ego a subi sans broncher la formation coranique puis l'apprentissage professionnel que son père lui a imposés, en dépit d'autres vocations) à un «bon fils " (l'aîné de sa descendance prend en charge matériellement une bonne part de la famille). Mais il se déroule dans une continuité qui n'est qu'apparente, au prix de sérieux raccourcis sur les ressentiments qu'a laissés la répudiation de l'épouse et de la mère. Il faudra un nouvel entretien dans la descendance du "vieux " pour la dévoiler, et pour comprendre le mérite du bon fils comme une forme de revanche à l'exemplarité sur les blessures imposées dans sa jeunesse par son père. Dans un autre cas, la démonstration de la soumission se déplace sur un père de substitution qui lui a offert «l'école jusqu'en $9^{e}$ année ». L'élève devenu adulte ne trouvera que des places de planton dans différents services administratifs, 
envol économique tronqué par la médiocrité des revenus et le coût des transports quotidiens vers des lieux de travail distants. La dette est pourtant payée par le fait que le nouveau logeur en puissance cherchera à ne pas s'éloigner du « grand frère » dans la capitale, en confiant à sa maisonnée l'organisation des baptêmes de ses propres enfants, c'est-à-dire en reportant sur lui le mérite de l'entregent et en faisant aveu du défaut d'argent pour ce faire.

Mais une obéissance ne fait pas les autres, comme le montre encore Mahalmoudou, un maçon reconverti en sous-traitant de bâtiment. Après qu'il ait montré tous les signes de la docilité dans sa jeunesse auprès du père, $\mathrm{y}$ compris en compromettant son début de carrière comme ouvrier qualifié, l'épreuve d'un isolement temporaire dans la capitale le fait monter en maturité : un cousin logeur conteste la femme qu'il a choisie et refuse désormais de le recevoir chez lui. Il faudra du temps pour faire admettre le mariage, quasiment le temps de sa rupture, et pour réassurer l'âné du respect du cadet. Longtemps après, c'est le tour de Mahalmoudou de déplorer la distance que deux jeunes parents qu'il a reçus ont prise avec lui, en lui portant contradiction sous son toit et en désavouant le contrôle matrimonial qu'il se devait de leur imposer : en réservant une épouse pour le premier, réfractaire à ce choix ; en dissuadant le divorce d'une seconde, déterminée à se rendre au tribunal et à ignorer la recommandation du chef de famille.

Mais sur la voie de la transgression et de la fuite de responsabilité, admise chez de jeunes garçons, les trajectoires ne divergent pas moins selon qu'elles sont suivies, ou non, du redressement attendu de conduite - ce sont les bêtises qui font bien grandir et de la capacité des hommes à pardonner les erreurs de leurs enfants. Un autre ressortissant de la région de Tombouctou, Mahamane, est devenu aménageur de jardins à Bamako. Sa maisonnée compte dix-sept personnes à l'enquête, conséquence de sept mariages dont le dernier seul reste actif, et de durables pratiques d'accueil. Mais son itinéraire personnel relie trois séries de fuite. L'une est d'abord imposée à son enfance par son propre père : il s'agit de se cacher pour échapper à l'école du colon en achetant la complicité du chef de canton. La deuxième est ensuite assumée par le jeune Mahamane qui s'évade à Bamako à l'insu de son père, puis transgresse l'autorité de son logeur à Bamako en trouvant emploi en reniant son nom de musulman. Cette logique d'émancipation le ramènera finalement et démonstrativement dans le droit chemin des valeurs familiales: paiement de l'impôt au village, envois réguliers de dons en nourriture, etc. La dernière « inconscience » est enfin reprochée à son propre fils, dont la cohabitation sans contribution incarne une forme contemporaine d'escapisme, et sur laquelle on reviendra plus bas.

D'un côté les fautes de jeunesse, dépassées par un surcroît d'exemplarité, celles du père, de l'autre la faute durable des jeunes, dans la vision d'un temps dégradé: le respect se perd. Rares sont les hommes dont les exigences se montrent plus variables, et renoncent à sur-imputer aux aînés la responsabilité à porter le nom du père. Pourtant, la chaîne des transmissions intergénérationnelle peut se trouver interrompue par de véritables ruptures de contrats éducatifs. L'obéissance n'est plus marquée ni dans l'immédiat ni de manière différée. Une décohabitation s'en suit brutalement car «on ne demande pas la route dans un conflit». C'est le cas d'un homme ayant d'abord reproché à son tuteur de ne pas s'être soucié de son mariage, qui exigeait constitution d'une dot, après qu'il lui ait fait «tous ses travaux au village ». C'est aussi le cas d'un père constatant que sa propre fille, détournée par « un inconnu 
avant même le mariage, refuse de revenir à la maison et a carrément foutu le camp de chez moi ».

\section{Cadet de fratrie, aînés de substitution : une inversion de rôle dans la migration}

-198 «jeunes » comptés parmi les descendances d'ego -, les enfants que les hommes ont reçus de leurs mariages sans les élever directement, ne sont qu'une poignée ; mais les enfants qu'ils ont hébergés significativement, sans être leurs pères, font un contrepoids notable (un tiers du groupe) à leurs enfants propres qu'ils ont élevés sous leur toit. Cadets dans leurs villages à quelques exceptions près, les migrants ont donc reçu plus d'enfants des autres qu'ils ont chargé ceux-ci des leurs. Ces enfants viennent d'ailleurs autant de frères et sœurs aînés que de plus jeunes. La dette familiale s'est donc doublement déplacée : vers un cadet et vers le citadin qu'il est devenu en prenant de l'âge. Il ne s'agit plus seulement de «financer un stock de grains, vêtir, payer l'impôt, contribuer à la dot" de parents au village, mais d'associer certains à l'environnement de la capitale, à des perspectives de travail rémunéré dans un marché $\mathrm{du}$ logement inaccessible aux villageois. Mais de frère à mari, de nouvelles contradictions se préparent pour la génération suivante.

\section{Frères et sœurs de même mère, enfants de même père}

$41 \mathrm{Au}$ Mali, l'impact de la distinction construite par la polygamie sur les figures du pouvoir, est en effet connue (Bagayoko 1980). Elle offre un schème récurrent de commentaire des affaires privées et publiques dans les différentes cultures ethniques du pays : d'un côté la solidarité, l'indulgence et l'affection sont volontiers exprimées entre frères et sœurs "même père même mère "; de l'autre, la rivalité plus ou moins 
refoulée, une émulation potentiellement agressive est « naturellement » attribuée aux enfants de plusieurs coépouses. La démarcation des deux filiations conduit à reprendre, à propos des migrants du Nord, les termes bambara de badenya pour la première et de fadenya pour la seconde.

Les hommes s'appuient sur ces termes pour décrire les contours changeants des maisonnées dans lesquelles ils ont d'abord été fils et frères, construisant graduellement leur responsabilité puis leur autorité d'hommes. L'inventaire des fratries se présente alors inégal : soigneusement individué, volontiers informé, assez précis sur les lieux de vie successifs de chacun, pour les membres de la badenya; flou, en particulier sur les filles, et souvent global, sans distinction de mères, pour les commentaires de la fadenya. Mais ce sont surtout les offres de tutorat et le traitement des besoins en hébergement qui distinguent les différents logements des hommes enquêtés. Autant la fratrie "même mère » entretient la circulation des enfants, à sens inégal certes, autant les obligations dues ou les initiatives prises à l'égard des frères et sœurs fadenya se présentent comme succinctes.

Le contraste de charge affective est frappant en particulier chez les plus gros hébergeants ayant reçu plus de personnes qu'ils n'en ont éduquées dans leur propre descendance. Soutien face aux épreuves de la vie, suivi des orientations prises ultérieurement à la résidence chez eux, l'empathie des hommes est durable. Elle vaut en particulier pour les enfants des sœurs, tandis que les vieux se montrent peu loquaces sur " les autres », nés de plusieurs mariages de leurs pères.

Aînées ou cadettes, les filles badenya profitent en particulier de la responsabilité d'hébergeant de leur frère. Non seulement ces sœurs reçoivent le gros des pratiques de confiage concernant des fillettes nées de leurs frères, le reste revenant à quelques mères divorcées selon des arbitrages citadins. Mais c'est surtout par leurs visites à Bamako que ces femmes libèrent une mobilité qu'elles ont rarement eue avec leurs maris. Ces mouvements vers la capitale et les échanges auxquels ils donnent lieu chez leurs frères sortent les femmes de formules stéréotypées sur l'immobilité et sur l'inactivité des ménagères, dont les hommes ne se privent pas quand ils parlent de leurs épouses. La sollicitude manifestée à l'égard de ces sœurs est appuyée, voire dépasse en nombre d'occasions et en souvenirs laissés, les expériences d'accueil de frères de même mère.

Mais une fois qu'ils sont devenus maris et pères, les hommes taisent la distinction des fratries dans leur propre descendance. Car l'autorité masculine se trouve quotidiennement à l'épreuve de la succession voire de la cohabitation des épouses et de leurs enfants, faute de leur offrir des maisons différentes. Les polygames réussissent donc plus ou moins bien à refouler les intérêts particuliers de la badenya au profit d'une fadenya consensuelle. Voir les filles quitter le domicile du père à son mariage est une règle dissymétrique transmise aux jeunes générations, mais arrangeante dans les difficultés économiques de la ville, car ces filles sont mariées plus tôt que leurs frères. Distinguer l'aîné des fils par un devoir d'exemplarité à "porter le nom du père " est une composante identitaire forte des gens du Nord. Mais assurer le même dû d'hébergement à tous les enfants constitue le paravent de la masculinité hégémonique : s'il convient de "montrer qui est le maître au-dessus des jalousies des femmes ", il s'agit surtout d'en contenir les risques et d'en dissimuler les débordements éventuels.

Les contradictions se dévoilent alors d'une génération à l'autre : quand «le sang est chaud à la maison", ou face à des défauts d'écoute engendrés par l'instabilité 
matrimoniale du père. Pourquoi ces fils nés à Bamako prendraient-ils en charge les " étrangers » de leur père-hôte qui en divorçant leur mère l'a laissée redevenir... une étrangère à la famille? Pourquoi s'investiraient-ils dans la réfection d'un bâtiment ou la montée en étage de la maison, au profit d'enfants nés d'autres femmes qui n'ont pas toujours été protectrices pour eux, quand leur propre entrée dans la vie adulte s'alourdit du coût du mariage et de difficultés d'insertion professionnelle ? Même si les "vieux » font encore semblant d'ignorer la brèche d'autorité qui suit des naissances très étalées et de porter haut et fier une fadenya respectueuse de leur autorité, ils sont conscients de ce que «chacun en ville connait d'où vient le bruit». C'est le cas avec Mahalmoudou qui, sur cinq mariages, en a conclu quatre par un divorce. Trois filles sur ses onze enfants ont grandi chez leurs mères; la dernière des fratries issues des unions malchanceuses se trouve même dissociée : la cadette n'est que suivie à distance chez sa mère, tandis que le fils reste sous le contrôle direct du père.

La perte de cohésion atteint enfin son point critique quand l'unique fils d'un premier mariage, vite et mal conclu, se trouve «perdu » - décès ou fuite migratoire - et n'est remplacé ni dans le mariage suivant ni par une incertaine expérience d'enfant confié.

\section{Changements et désordres : filles méritantes, fils désavoués}

Sous ajustement et en individuation, la masculinité ne peut faire fi, enfin, de pratiques bonifiantes venues de jeunes femmes, ou de nouvelles performances pouvant menacer à terme les monopoles économiques des hommes. Dans les remous de l'hébergement, quelques pères évoquent ainsi deux figures minoritaires mais inverses sur le plan du mérite et du démérite particuliers dans la descendance. Tant la distinction de certaines filles que la déconsidération de fils, dont on attendait précisément trop, touchent au vif la cohabitation des sexes. Dans les mêmes voisinages, la satisfaction qu'inspirent des filles retardant leur décohabitation rencontre donc des reproches publics adressés à des garçons déjà mariés qui prolongent leur cohabitation en des termes abusifs.

\section{De « bonnes filles » à " filles intelligentes »}

Les bonnes filles sont à l'image des bonnes mères : elles prennent les enfants qu'on veut bien leur confier; elles sortent de leur famille en suivant leurs maris mais en continuant d'adresser une reconnaissance symbolique à leur père: l'une prépare et envoie un plat de cuisine, l'autre «ne peut pas faire deux ou trois jours sans passer ici ", une autre encore se fait accompagner de son mari pour remettre sa première paye au précédent tuteur.

De nouveaux mérites pointent cependant dans les commentaires des hommes. Ils ne tiennent pas tant à la fréquentation scolaire, qui bien que redressée depuis la Troisième République reste discriminée par le sexe des élèves (Lange 1998), qu'à des surperformances remarquées à l'égard de frères voire de maris. L'investissement scolaire des filles est alors chargé de nouvelles potentialités: non pas assurer les futures ressources d'un ménage, comme il revient aux garçons de le faire, ni même rééquilibrer les contributions entre époux, qui restent secrètes, mais " aider le pays " ou «faire quelque chose pour notre région avec tous ses problèmes ». Cette dédomestication de futures participations féminines est à souligner, les hommes eux ne 
s'étant pas montrés très performants sur la question du développement. De ce fait, une figure féminine de modernité pourrait émerger au même titre que la sensibilisation à la contraception (Dialmy 2009), sans attaquer de front la masculinité hégémonique et plutôt en lui retournant une dette d'origine.

Le changement est en effet valorisé par les hommes plus souvent que leurs épouses dans le milieu populaire considéré. Il leur en coûte doublement: par la charge de l'investissement scolaire, et par le retard de décohabitation qui se profile par rapport aux sœurs mariées tôt. Ces cas restent pour l'heure atypiques dans le groupe enquêté et ne privent pas la majorité des commentaires des stéréotypes habituels sur les filles qui ne font « que des bricoles ». Il n'empêche qu'à défaut d'être déjà payant, le changement est amorcé et pourrait se confirmer par la comparaison de deux générations de filles que mène Mahalmoudou :

«Ma petite sœur qui est décédée cette année, elle avait carrément perdu ses études

à Tombouctou, parce qu'on l'a mise dans le mariage en 88. C'est comme une trahison puisque nos oncles de Tombouctou ont donné sa main à un monsieur de Bamako, sans notre accord, alors qu'elle était en huitième année. Donc, elle n'a pas pu poursuivre. Elle s'est mariée avec un cuisinier sans étude! Celui-là, il a même peur qu'elle progresse, il l'a fait rester ménagère. [...] Parmi mes filles y a Fadimata qui est de 1977. Elle a fait les quatre ans à l'université. Elle a même fini avec la soutenance. Donc maintenant, elle attend le boulot. C'est pas par manque de mari, mais elle se donne à l'étude, pour finir. C'est l'idée qu'elles avaient mes filles, il faut étudier. Elles ne veulent pas se marier tôt comme leurs mamans. Sa sœur Niafata a fait l'université aussi. Elles sont très intelligentes ces femmes. Kangaï elle est née en 1982, chez moi à Sikoro, elle n'est pas mariée non plus. Elle est à l'école de santé dans sa dernière année, elle fait l'examen pour être infirmière. J'ai eu la chance. Mais je vais prendre encore une quatrième année pour qu'elle soit sage-femme. Je vais me forcer, ça vaut le coup : sage-femme, c'est au-dessus d'infirmière. »

Le changement est d'autant plus notable que les pères ont coutume d'interpeller leurs fils comme "vauriens », entre autres sur le plan scolaire, pour prévenir ou désamorcer tout reproche au favoritisme. Le risque est alors de placer ces filles assidues en nouvelles concurrences avec les hommes de leur génération : sur le marché du travail, sur le terrain domestique, car il faudra alors héberger « utile à leurs métiers» en les soulageant des tâches ménagères, voire sur le marché des terrains et pour sécurisation économique de la maison, comme c'est déjà le cas dans les milieux plus nantis de la capitale malienne. Le pari est aussi de les pousser vers de meilleurs mariages que ceux de la plupart des filles et des sœurs, avec effets de retour possible sur le nom du père.

\section{Dénigrements entre hommes : vers un reniement masculin ?}

Face aux bons fils capables de maintenir le nom du père par-delà les tensions familiales, d'autres fils aînés, devenus pères à leur tour, font l'objet de désapprobations ouvertes dans les voisinages et se retrouvent désignés comme n'étant " pas à la hauteur » : ni des transmissions intergénérationnelles ni des contributions attendues dans la vie urbaine. La marginalisation de la masculinité se vit donc au sein de la maison, avec des cohabitations rendues impossibles quand les autres frères et sœurs sont pris à témoins de la brebis galeuse. Dans un cas, la honte ressentie par le père à l'égard de son fils de vingt-huit ans se traduit par un commentaire sec en direction de la chambre occupée par le jeune ménage :

«Ses deux gosses ont quatre et un an. Ils sont nés chez moi, ils vivent ici. Aucun

autre de mes garçons [7 sur un total de 11 enfants nés d'un seul mariage] après lui 
n'est marié. Il a fait son école ici. Après il a abandonné pour le commerce. C'est moi qui l'héberge, moi qui paye pour son impôt. Et lui il ne donne rien pour la dépense de jour.»

Il faudra prolonger l'entretien pour comprendre que ce vendeur ambulant dans la capitale a connu un meilleur destin de transitaire entre Bamako et l'intérieur du pays pour le commerce du mil. Le déclin de ses activités est attribué au fait qu'il n'a ni présenté de rentrées financières au père, ni amorcé de participation aux charges de la famille, dans une formule d'hébergement que le « vieux » avait pourtant concentrée sur sa descendance directe.

5 Le second cas met plus durement en péril l'ordre de la cohabitation, puisque « l'égoïsme » de l'aîné des garçons interpelle des réseaux sociaux plus larges, au village et parmi des collègues en ville. Il s'agit du seul enfant de la première épouse de Mahamane, divorcée, le premier de douze autres dans la maison, et à quarante ans le témoin de l'histoire prodigue de son père et d'un hébergement particulièrement performant.

56 L'inventaire paternel est en effet divers en générations d'hébergés et origines des visiteurs. Son sens du sacrifice se mesure en nombre de personnes reçues, en nombre d'années passées à Bamako, "jusqu'au mariage ", en entregents mobilisés au Mali : professionnels pour négocier des emplois arrangeants, communautaires pour arranger diverses cérémonies. Il se montre cumulatif à tous égards : formation de jeunes, accueil régulier d'actifs saisonniers, de malades et d'orphelins, dans les parentés alliées et directes, depuis le village d'origine, ses alentours et l'étranger, en maintenant la fonction de logeur pour les enfants des premiers logés. Cousins, élèves, gens de caste, tous y passent une fois la propriété assurée à Bamako.

57 Le ton change pourtant pour l'hébergement qui devrait être le plus légitime, promesse déçue de relai :

«Faire le logeur comme ça, je ne pense pas que mes enfants seront capables. Je l'ai dit devant mon premier garçon [40 ans]. Normalement il habite dans ma maison, il doit contribuer. Mais il ne le fait pas. J'ai même décidé de le faire sortir d'ici, mais les hommes [de la grande famille] m'ont prié de le laisser rester. Sinon il travaille! Même les gens avec lesquels il travaille, ils payent leur location, ils donnent leurs condiments [contribution quotidienne à la cuisine]. Et lui ici, il contribue rien! Il est marié, c'est moi qui élève ses [3] enfants! Son petit a sept ans, il vient de rentrer à l'école. Il paye rien! Il paye pas le prix de condiment, il achète pas le mil, il paye pas le courant! J'ai dit ça! Avec ta conscience, tu es dans une maison, tu manges, tu bois, tu utilises les WC, tu manges le matin, tu manges le repas de journée, tu manges le repas de nuit : tu fais rien ? Il dit qu'il ne gagne pas de l'argent. C'est pas vrai ! C'est moi qui l'ai fait devenir jardinier. Un garçon comme ça si tu le mets dans la famille, il fera des histoires avec ses frères! Ils vont faire des histoires, parce que c'est pas ses frères et ses sœurs qui va le faire nourrir, lui et ses enfants encore! Moi à mon âge, j'ai vingt personnes qui habitent ici, personne ne paye de location, personne! Avec mon métier, on me donnait 50000 francs par mois. Ce n'est même pas un prix de condiment! Les mamans qu'est-ce qu'elles vont dire? Non, les gens quand ils sont inconscients il faut les foutre dehors pour qu'ils puissent comprendre! Moi j'ai un ami, il a ses enfants qui travaillent, y a ses enfants qui achètent les draps, sa femme qui achète les rideaux, ils contribuent tous. Bon, y a un enfant qui est ingénieur, il participe pas. Il l'a mis dehors ! Tellement moi j'ai fait pour les parents, alors que ce fils-là ne fait rien. »

Fiertés exprimées et hontes retenues, trahisons et reconnaissances, confrontations et fuites s'adossent donc entre elles dans ces pratiques et représentations de 
l'hébergement. Quand la composition sociologique et les conditions matérielles de l'habitation changent, c'est moins par réfutation de normes que par chassés-croisés de mouvements résidentiels et par bifurcation de conduites dans les relations intergénérationnelles. Le contrôle du sol, de l'employabilité, du mariage des cadets, se joue bel et bien en ville depuis la gestion d'une maison et ses corollaires, la mobilité imposée et la décohabitation acceptée. Force est de constater que l'autorité des hommes s'effrite en la matière, au fur et à mesure que les migrants les moins qualifiés se déclassent dans les périphéries urbaines.

L'accueil de nouveaux dépendants familiaux différencie pourtant sensiblement les parcours d'hommes dans un échantillon constitué a priori sur des bases homogènes. Ils révèlent une masculinité à plusieurs visages : selon les moyens dont disposent ceux qui se disent désormais " chefs de ménage " pour accueillir parents et étrangers ; selon les capacités, entendues sous un double jour économique et moral, qui démarquent sensiblement les propriétaires des locataires; selon les sollicitations qui différencient badenya et fadenya; et selon la génération qui distingue anciens et nouveaux citadins dans leurs moments propres de passage à l'âge adulte. Face à des hommes encore passeurs de références villageoises, s'étiolent les dettes migratoires, se retravaillent les contrats éducatifs, se renégocient les rapports entre les sexes. À l'épreuve de la grande ville, ces cohabitations en transition n'échappent ni à l'individuation des responsabilités ni à une reconsidération des qualités masculines au regard de possibles émancipations féminines.

L'approche qualitative permet ainsi de contrôler les effets sur la masculinité : de l'âge dans l'avancée du cycle de vie, de la génération dans les repositionnements géographiques d'une grande famille, et de conjonctures historiques dans un temps déroulé $\mathrm{du}$ "troisième âge » colonial à la «lutte contre la pauvreté » du millénaire naissant. Dans ces termes plus analytiques que symboliques, le devenir des hommes ne peut que renvoyer à une perspective plurielle : travailler une ligne de conduite pour se montrer à la hauteur des circonstances, mais pour aussi intégrer les changements de rôle vécus dans les mêmes temps par les femmes, mères et filles.

\section{BIBLIOGRAPHIE}

ANTOINE, P. (DIR.)

2007 - Les relations intergénérationnelles en Afrique. Approche plurielle, Paris, Centre Population et Développement, Rencontres.

BAGAYOKO, S.

1980 - « Lieux et théorie du pouvoir dans le monde mandé : passé et présent », Cahiers des Sciences humaines, 25 (4) : 445-460.

BERTRAND, M.

2001 - « Femmes et marchés fonciers urbains : mesures et déterminants d'une percée à Bamako, Mali », Autrepart, 19 : 29-48. 
2004 - « Femmes et modernité citadine au Mali », in S. DENÈFLE (dir.), Femmes et villes, Tours, Presses universitaires François-Rabelais : 283-304.

2011a - De Bamako à Accra. Mobilités urbaines et ancrages locaux en Afrique de l'Ouest, Paris, Karthala. $2011 \mathrm{~b}$ - «"Quand femme rime avec local" : logique de promotion ou nouveau confinement dans les villes africaines? », Justice spatiale/ Spatial justice, 3, <http://www.jssj.org>.

BoUju, J.

2009 - « La malédiction, l'honneur et la spéculation », Bulletin de l'APAD, 29-30, <http:// apad.revues.org/4006>.

BROQUA, C. \& ЕвоKO, F. (DIR.)

2009 - « La fabrique des identités sexuelles », numéro spécial, Autrepart, 1 (49).

CONNELL, R. W.

1995 - Masculinities, Los Angeles, University of California Press.

DIALMY, A.

2009 - Vers une nouvelle masculinité au Maroc, Dakar, Codesria.

DUMESTRE, G. \& TOURÉ, S.

1998 - Chroniques amoureuses au Mali, Paris, Karthala.

GIBBAL, J.-M.

1974 - Citadins et villageois dans la ville africaine. L'exemple d'Abidjan, Paris, Maspero.

GORMAN-MURRAY, A.

2008 - « Masculinity and the Home : A Critical Review and Conceptual Framework », Australian Geographer, 39 (3) : 367-379.

JAMIN, P.

2003 - « Vivre ensemble ou la douleur d'être "en grande famille" », Politique Africaine, 91 : 33-50.

LANGE, M.-F. (DIR.)

1998 - L'école et les filles en Afrique. Scolarisation sous conditions, Paris, Karthala.

MORRELL, R. (ED.)

1998 - « Masculinities in Southern Africa », Special Issue, Journal of Southern African Studies, 24 (4).

OLIVIER DE SARDAN, J.-P.

1984 - Les sociétés songhaï-zarma (Niger-Mali). Chefs, guerriers, esclaves, paysans..., Paris, Karthala.

OPPONG, C.

2006 - « Familial Roles and Social Transformations. Older Men and Women in Sub-Saharan

Africa », Research on Aging, 28 (6) : 654-668.

OUÉDRAOGO, D. \& PICHÉ, V. (DIR.)

1995 - L'insertion urbaine à Bamako, Paris, Karthala.

ROTH, C.

2007 - «"Tu ne peux pas rejeter ton enfant !" Contrat entre les générations, sécurité sociale et vieillesse en milieu urbain burkinabè », Cahiers d'Études africaines, XLVII (1), 185 : 93-116.

VUARIN, R.

1994 - « L'argent et l'entregent », Cahiers des Sciences humaines, 30 (1-2) : 255-273. 


\section{NOTES}

1. "Critical Perspectives on Working with Men and Masculinities to Promote Empowerment for Girls and Women", EADI/DSA International Conference « Rethinking Development in an Age of Scarcity and Uncertainty. New Values, Voices and Alliances for Increased Resilience ", York, University of York, 19-22 September 2011.

2. Leurs défaillances respectives sont inégalement consignées dans les «secrets du mariage " (ROTH 2007) : tandis que l'incapacité d'un mari devra rester tue, celle d'une femme peut être rendue publique du jour au lendemain.

3. «La proportion d'hommes qui arrivent à Bamako à titre d'hébergés décroît avec l'âge... Chez les femmes, cette proportion d'hébergées est toutefois relativement stable et généralement supérieure à $95 \%$ quel que soit l'âge à l'arrivée » (OUÉDRAOGO \& PICHÉ 1995 : 101).

4. Des natifs de l'Ouest du Mali offrent un second groupe d'enquête au Projet Mobilités ouest-africaines financé par le Programme ANR-AIRD «Les Suds ». Les « gens du Nord » ont été visités dans deux quartiers périphériques urbanisés au moment où ces ressortissants montaient en nombre dans la population de Bamako, depuis les sécheresses répétées des années 1970-1980.

5. Ils comptent en moyenne quatorze personnes dans la génération considérée. Les plus petits relèvent d'un défaut de moyens économiques plus que d'un choix délibéré de restreindre la descendance. Les plus chargés multiplient les formes d'hébergement temporaire.

6. Sur les quarante unions enregistrées par l'échantillon des quinze hommes, il ne reste plus que dix-neuf mariages actifs en fin de vie. Les migrants devenus citadins ont perdu leurs épouses plus souvent par séparation que par décès.

7. Le terme « malo » est celui qui exprime la réserve et la bonne tenue dans les relations sociales, la capacité d'opposer du sang-froid aux mauvais emportements, aux paroles et comportements trop directs.

\section{RÉSUMÉS}

$\mathrm{Au}$ regard d'approches intéressant au Mali le sujet collectif «femmes», les rapports intergénérationnels offrent des pistes heuristiques pour graduer, dans la capitale, les masculinités d'hégémoniques à marginalisées. Ce texte s'attache à des capacités sociales fortement revendiquées par les hommes : leur vocation à «héberger » étrangers, épouses et descendances, parentés directe et indirecte. L'honneur de l'hôte se défend dans l'installation d'une maison et le maintien de cohabitations résidentielles, dont il assume la charge et déplore les heurts, pour tenir rang dans la vie urbaine. À différentes étapes de mobilisation et de déstabilisation de références normatives, les rôles s'inversent dans l'hébergement; des contradictions se dévoilent ; les mérites reconnus à certains rencontrent les reproches adressés à de nouvelles générations d'hommes pour la redistribution des ressources urbaines. Plus 
individuées, ces visions masculines contextualisent les rapports de genre dans les termes d'une transition migratoire, démographique et métropolitaine.

Sons, Brothers, Fathers

Since "women" tend to be analysed as a collective subject in Mali, the intergenerational relationships offer heuristic ways to evaluate the masculinity in a range of hegemonic marginalised positions, in the capital of the country. This paper deals with social capacities which are still strongly asserted by men : their authority in the matter of "lodging" as much as possible of strangers, wives and descendants, from direct and indirect kinships. The honour of the logeur is defended through the setting of a house and the ability to support co-dwellers on its name, assuming the load and clashes of a family to gain prestige in the urban life. Normative references are sometimes mobilised sometimes destabilised in the course of men's life : housing practices can reverse the social roles, or reveal new contradictions ; merits recognized for some family members can meet reproaches addressed to new generations of men concerning the redistribution of urban resources. These viewpoints on male duties tend to be more individual, but they put gender relationships in the prospect of a triple transition : migratory, demographic and metropolitan.

INDEX

Mots-clés : Mali, masculinité, migration, parenté, rapports intergénérationnels, urbanité Keywords : Mali, Masculinity, Migration, Kinship, Intergenerational Relationships, Urbanity

\section{AUTEUR}

\section{MONIQUE BERTRAND}

Développement et Sociétés (UMR 201), IRD ; Université de Paris 1, Paris. 